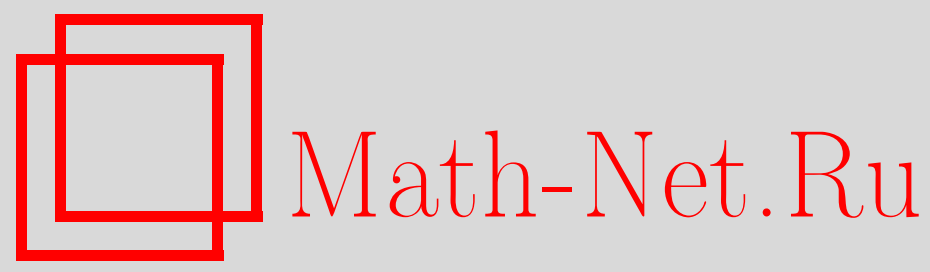

А. Г. Брусенцев, Многомерная теорема Г. Вейля и накрывающие семейства, Матем. заметки, 2003, том 73, выпуск $1,38-48$

DOI: https://doi.org/10.4213/mzm166

Использование Общероссийского математического портала Math-Net.Ru подразумевает, что вы прочитали и согласны с пользовательским соглашением http://www . mathnet.ru/rus/agreement

Параметры загрузки:

IP : 52.205 .19 .152

26 апреля 2023 г., 02:33:30

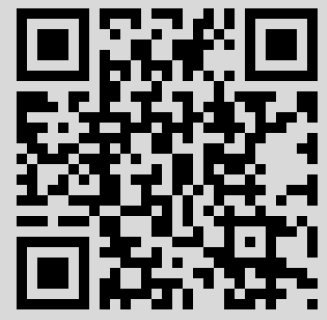




\section{МНОГОМЕРНАЯ ТЕОРЕМА Г. ВЕЙЛЯ И НАКРЫВАЮЩИЕ СЕМЕЙСТВА}

\section{А. Г. Брусенцев}

Получено обобщение на эллиптические операторы второго порядка в $L_{2}(G)$ $\left(G \subseteq \mathbb{R}^{n}\right)$ известной теоремы $\Gamma$. Вейля о самосопряженности в существенном оператора Штурма-Лиувилля $L u=-\left(p(x) u^{\prime}\right)^{\prime}+q(x) u, D_{L}=C_{0}^{\infty}\left(\mathbb{R}^{1}\right)$ в $L_{2}\left(\mathbb{R}^{1}\right)$ при $p(x)>0, q(x) \geqslant$ const. Многомерная теорема Вейля выводится из более общей теоремы, для формулировки и доказательства которой строится особая конструкция накрывающего семейства. Установленные резултаты содержат известные многомерные аналоги теоремы Вейля и, в отличие от них, относятся к области $G$, которая может быть собственным подмножеством $\mathbb{R}^{n}$.

Библиографиял: 9 названий.

1. Введение. В настоящей работе рассматривается вопрос о самосопряженности в существенном эллиптического оператора в $L_{2}(G)$, имеющего вид

$$
M u=(\nabla-i \vec{b}(x))^{*}(A(x)(\nabla-i \vec{b}(x)) u)+q(x) u,
$$

$D_{M}=C_{0}^{\infty}(G)$. Здесь $G$-открытоемножество в $\mathbb{R}^{n}, A(x)$ - позитивная симметрическая (вещественная) матрица-функция, $\vec{b}(x)-n$-компонентная вектор-функция с вещественными компонентами, а $q(x)$ - вешественная функция, удовлетворяющая условию

$$
q(x) \geqslant \text { const, } \quad x \in G \text {. }
$$

Известная теорема Вейля [1] равносильна тому, что оператор Штурма-Лиувилля

$$
L u=-\left(p(x) u^{\prime}\right)^{\prime}+q(x) u,
$$

$D_{L}=C_{0}^{\infty}\left(\mathbb{R}^{1}\right)$ с достаточно гладкими функциями $p(x)>0, q(x) \geqslant$ const самосопряжен в существенном в пространстве $L_{2}\left(\mathbb{R}^{1}\right)$. Примеры, построенные в работах [2], [3], показывают, что при $G=\mathbb{R}^{n}$ и $n \geqslant 3$ оператор $M$ вида (1), (2) может иметь ненулевые индексы дефекта даже при $\vec{b}(x)=\overrightarrow{0}, q(x)=$ const. С другой стороны, работы [4], [5] содержат многомерные аналоги теоремы Вейля для матрищ $A(x)$ некоторых частных видов. Настоящая работа посвящена расширению класса матриц $A(x)$, для которого справедливо обобщение теоремы Вейля. При этом наш класс матриц не только содержит уже описанные виды, но, в отличие от них, относится к области $G \subseteq \mathbb{R}^{n}$, которая может быть собственным подмножеством $\mathbb{R}^{n}$.

В работе многомерная теорема Вейля (теорема 1) выводится из более общей теоремы 2 , для установления которой развит особый метод накрывающих семейств, подсказанный оригинальным приемом работы [4].

Ниже через $(\cdot, \cdot),|\cdot|$ обозначается скалярное произведение и норма в унитарном пространстве $E(\operatorname{dim} E<\infty)$, а через $\langle\cdot, \cdot\rangle,\|\cdot\|$ - скалярное произведение и норма в бесконечномерном пространстве. 
2. Локальные условия на коэффициенты оператора $M$. Обозначим через $\operatorname{Lip}_{\alpha \text { loc }}(G)$ множество функций $f(x)$, определенных в $G$ и удовлетворяющих при некотором $\alpha \in(0 ; 1]$ условию

$$
\left|f\left(x_{0}+y\right)-f\left(x_{0}\right)\right|=O\left(|y|^{\alpha}\right) \quad \text { при }|y| \rightarrow 0
$$

для любой точки $x_{0} \in G$. При этом константа в $O(\cdot)$, вообще говоря, зависит от $x_{0}$. Ниже через $C^{(1, \alpha)}(G)$ обозначается множество функций из $C^{1}(G)$, частные производные которых принадлежат $\operatorname{Lip}_{\alpha \text { loc }}(G)$.

У всякой функции $f(x) \in \operatorname{Lip}_{1 \text { loc }}(G)(\alpha=1)$ почти всюду в $G$ существуют частные производные первого порядка (см. [6, с. 295]). Поэтому оператор $M$ можно считать корректно определенным на $C_{0}^{\infty}(G)$ при условиях

$$
a_{i j}(x), b_{j}(x) \in \operatorname{Lip}_{1 \mathrm{loc}}(G), \quad q(x) \in L_{2 \text { loc }}(G)
$$

Обозначим через $D_{\text {loc }}\left(M^{*}\right)$ множество функций $u \in L_{2}$ loc $(G)$, для каждой из которых найдется такая функция $g \in L_{2 \text { loc }}(G)$, что и при всех $\phi \in C_{0}^{\infty}(G)$ справедливо равенство

$$
\langle u, M \phi\rangle=\langle g, \phi\rangle
$$

где $\langle\cdot, \cdot\rangle$ - скалярное произведение в $L_{2}(\operatorname{supp} \phi)$. В частности,

$$
D_{M}{ }^{*} \subset D_{\mathrm{loc}}\left(M^{*}\right)
$$

В дальнейшем мы предполагаем коэффициенты выражения $M$ имеющими такие локальные свойства, что выполнено

УСЛовИЕ А. Для всякой функиии $u \in D_{\mathrm{loc}}\left(M^{*}\right)$ и любой ограниченной области $\Omega(\bar{\Omega} \subset G)$ существует последовательность $\left\{u_{m}\right\}_{m=1}^{\infty}$ функиий из $C^{2}(G)$, для которой $u_{m} \rightarrow u$ в $L_{2}(\Omega)$ u

$$
\lim _{m \rightarrow \infty} \operatorname{Re}\left\langle\psi M u_{m}, u_{m}\right\rangle_{L_{2}(\Omega)}=\operatorname{Re}\langle\psi g, u\rangle_{L_{2}(\Omega)}
$$

при любой вещественной функиии $\psi \in C_{0}(\Omega)$.

Приведем явные достаточные условия реализации условия А.

ПРЕДЛОЖЕНИЕ. Пусть в операторе $M$ вида (1)

$$
a_{i j}(x) \in C^{(1, \alpha)}(G), \quad b_{j}(x) \in C^{1}(G), \quad \alpha \in(0,1]
$$

а функиия $q(x) \in L_{2 \operatorname{loc}}(G)$ и, вместо (2), локально ограничена снизу. Тогда условие $A$ выполнено. 
ДоКАЗАТЕЛЬСТВО. По оператору $M$ и ограниченной области $\Omega$ построим оператор $M_{\Theta}$, действующий в пространстве $L_{2}\left(\mathbb{R}^{n}\right)$. Пусть $\Omega_{0}, \Omega_{1}$ - такие ограниченные открытые множества, что

$$
\bar{\Omega} \subset \Omega_{0}, \quad \bar{\Omega}_{0} \subset \bar{\Omega}_{1}, \quad \bar{\Omega}_{1} \subset G .
$$

Обозначим через $A_{1}(x), \vec{b}_{1}(x), q_{1}(x)$ функции, определенные в $\mathbb{R}^{n}$, совпадающие с $A(x)$, $\vec{b}(x)$ и $q(x)$ на $\Omega_{1}$ и равные нулю вне $\Omega_{1}$. Пусть $0 \leqslant \Theta(x) \leqslant 1-$ функция из $C^{\infty}\left(\mathbb{R}^{n}\right)$, причем $\Theta(x) \equiv 1$ при $x \in \Omega_{0}, \Theta(x) \equiv 0$ при $x \notin \Omega_{1}$. Рассмотрим в $L_{2}\left(\mathbb{R}^{n}\right)$ оператор

$$
M_{\Theta} u=\left(\nabla-i \Theta \vec{b}_{1}(x)\right)^{*}\left(A_{\Theta}(x)\left(\nabla-i \Theta \vec{b}_{1}(x)\right) u\right)+\Theta q_{1}(x) u
$$

$D_{M_{\Theta}}=C_{0}^{\infty}\left(\mathbb{R}^{n}\right)$. Здесь $A_{\Theta}(x)=\Theta(x) A_{1}(x)+(1-\Theta(x)) I_{n}, I_{n}$ - единичная $n \times n$ матрица; $M_{\Theta}$ - эллиптический оператор, удовлетворяющий всем условиям теоремы 1 из работы [4]. В силу этой теоремы он является самосопряженным в существенном. Пусть $u(x) \in D_{\text {loc }}\left(M^{*}\right)$. Тогда при $x \in \Omega g=M u=M_{\Theta} u$. Пусть функция $\phi(x) \in C_{0}^{\infty}\left(\mathbb{R}^{n}\right)$, $\phi(x) \equiv 1$ при $x \in \Omega$ и $\phi(x) \equiv 0$ при $x \notin \Omega_{0}$. Если $u_{1}=u$ при $x \in \Omega_{0}$ и $u_{1} \equiv 0$ при $x \in \mathbb{R}^{n} \backslash \Omega_{0}$, то функция $\phi u_{1} \in L_{2}\left(\mathbb{R}^{n}\right)$. Воспользовавшись адаптацией метода Като [7], получаем, что $\phi u_{1} \in D_{M_{\Theta}^{*}}=D_{M_{\Theta}}$. Поэтому существует последовательность $\left\{u_{m}\right\}$ элементов $C_{0}^{\infty}\left(\mathbb{R}^{n}\right)$ такая, что $u_{m} \rightarrow \phi u_{1}$ и $M_{\Theta} u_{m} \rightarrow M_{\Theta}\left(\phi u_{1}\right)$ в $L_{2}\left(\mathbb{R}^{n}\right)$. Поскольку при $x \in \Omega$

$$
M_{\Theta}\left(\phi u_{1}\right)=M u=g, \quad M_{\Theta} u_{m}=M u_{m},
$$

получаем $u_{m} \rightarrow u, M u_{m} \rightarrow g$ в $L_{2}(\Omega)$. Отсюда следует справедливость условия А. Предложение доказано.

3. Теорема Вейля для многомерного случая. Рассмотрим область вида

$$
G=G_{1} \times G_{2} \times \cdots \times G_{k}, \quad k \geqslant 1,
$$

где открытые множества $G_{j} \subseteq \mathbb{R}^{n_{j}}\left(\sum n_{j}=n\right)$. При $x \in G$ будем писать $x=\left\{\vec{x}_{1}, \vec{x}_{2}\right.$, $\left.\ldots, \vec{x}_{k}\right\}$, где $\vec{x}_{j} \in G_{j}$. Основные ограничения относятся к множеству $G \backslash Q$, где область

$$
Q=Q_{1} \times Q_{2} \times \cdots \times Q_{k}
$$

а $Q_{j}$ - ограниченные открытые множества в $\mathbb{R}^{n_{j}}$ такие, что $\bar{Q}_{j} \subset G_{j}$.

Ниже для функции $f\left(\vec{x}_{j}\right)$, определенной в $G_{j} \backslash Q_{j}$, условие $f\left(\vec{x}_{j}\right) \rightarrow \infty$ при $\vec{x}_{j} \rightarrow \partial G_{j}$ означает, что для любого $N>0$ в $G_{j}$ существует такой компакт $R_{N}\left(\bar{Q}_{j} \subseteq R_{N}\right)$, что при $\vec{x}_{j} \in G_{j} \backslash R_{N}$ выполнено неравенство $\left|f\left(\vec{x}_{j}\right)\right|>N$.

ТЕОРема 1. Пусть для определенной в $G$ вида (4) симметрической позитивной матрицы-функиии $A(x)$ найдется такая область $Q$ вида (5), что при $x \in G \backslash Q$ $A(x)$ имеет блочно-диагональный вид

$$
A(x)=\operatorname{diag}\left\{a_{j}\left(\mu_{j}\left(\vec{x}_{j}\right)\right) B_{j}\left(\vec{x}_{j}\right)\right\}_{j=1}^{k},
$$

где $\vec{x}_{j} \in G_{j} \backslash Q_{j}, a_{j}(\cdot)$ - определенные в $\mathbb{R}^{1}$ положсительные функиии из $C^{1}\left(\mathbb{R}^{1}\right)$, $\mu_{j}\left(\vec{x}_{j}\right)$ - такие функиии из $C^{(1,1)}\left(G_{j} \backslash Q_{j}\right)$, ито $\left|\nabla \mu_{j}\right|>0 u$

$$
\mu_{j}^{2}\left(\vec{x}_{j}\right) \rightarrow \infty \quad \text { npu } \quad \vec{x}_{j} \rightarrow \partial G_{j}
$$


а $B_{j}\left(\vec{x}_{j}\right)$ - матрицы-функиии размера $n_{j} \times n_{j}$ с әлементами из $\operatorname{Lip}_{1 \text { lос }}\left(G_{j} \backslash Q_{j}\right)$, удовлетворяющие неравенствам

$$
\begin{gathered}
\nabla\left(B_{j} \nabla \mu_{j}^{2}\right) \geqslant 0 \quad \text { почти всюду в } G_{j} \backslash Q_{j} \\
\left(B_{j} \nabla \mu_{j}, \nabla \mu_{j}\right) \leqslant \mathrm{const}, \quad \vec{x}_{j} \in G_{j} \backslash Q_{j} .
\end{gathered}
$$

Тогда при выполнении условий (3) и условия $A$ оператор $M$ вида (1), (2) с такой матричей $A(x)$ самосопряжен в существенном в пространстве $L_{2}(G)$.

Отложив доказательство теоремы 1 до п. 4, рассмотрим простейшие следствия из нее.

СлЕДСТВИЕ 1. Пусть $G=\mathbb{R}^{n}$. Если в операторе $M$ матрица $A(x)$ nрu $|x| \geqslant$ $N>0$ имеет вид

$$
A(x)=\operatorname{diag}\left\{a_{1}\left(x_{1}\right), a_{2}\left(x_{2}\right), \ldots, a_{n}\left(x_{n}\right)\right\},
$$

әде $a_{j}(\cdot)$ - положительные функиии одной переменной из $C^{1}\left(\mathbb{R}^{1}\right)$, то при выполнении условий (3) и условия $A$ оператор $M(1),(2)$ самосопряжен в существенном.

ДокАЗАТЕЛЬСТво состоит в непосредственной проверке условий теоремы $1 \mathrm{c} G_{j}=$ $\mathbb{R}^{1}\left(n_{j}=1\right)$ и функциями $\mu_{j}\left(x_{j}\right)=x_{j}$, а также $(1 \times 1)$-матрицами $B_{j}\left(x_{j}\right)=1$.

Отметим, что частньй случай следствия 1 с четньми функциями $a_{j}(\cdot)$ и с условиями гладкости предложения из п. 2 сформулирован без доказательства в работе [4]. В этой же работе при тех же условиях гладкости установлена самосопряженность в существенном оператора $M(1),(2)$ с матрицами следующих двух видов:

$$
A(x)=a(|x|) B_{0}, \quad A(x)=a(|x|) \beta\left(\frac{x}{|x|}\right) I_{n},
$$

где $a(\cdot), \beta(\cdot)$ - положительные функции соответственно на $[0, \infty)$ и единичной сфере в $\mathbb{R}^{n}$, а $B_{0}$ - постоянная симметрическая позитивная матрица. Этот результат является частным случаем теоремы 1 при $k=1, G_{1}=G=\mathbb{R}^{n}$ с $\mu_{1}\left(\vec{x}_{1}\right)=\mu(x)=|x|$, $B_{1}\left(\vec{x}_{1}\right)=B(x)=B_{0}$ в первом случае и $B(x)=\beta(x /|x|) I_{n}-$ во втором. Действительно,

$$
\begin{gathered}
\nabla\left(B_{0} \cdot \nabla|x|^{2}\right)=2 \operatorname{Sp} B_{0}>0, \\
\nabla\left(\beta\left(\frac{x}{|x|}\right) I_{n} \cdot \nabla|x|^{2}\right)=2(\nabla \beta, x)+2 n \beta=2 n \beta\left(\frac{x}{|x|}\right)>0 .
\end{gathered}
$$

Поэтому в обоих случаях выполнено (8). Условие же (9) в обоих случаях очевидно.

СлЕДСТВИе 2. Пусть $G=\mathbb{R}^{n}$. Если матрица $A(x)$ в операторе $M$ nрu $|x| \geqslant$ $N>0$ имеет вид

$$
A(x)=a(\mu(x)) I_{n},
$$

әде $0<a(\cdot) \in C^{1}\left(\mathbb{R}^{1}\right)$, функиия $\mu(x) \in C^{2}\left(\mathbb{R}^{n} \backslash\{x:|x|<N\}\right)$ такова, что $\mu^{2}(x) \rightarrow \infty n p u|x| \rightarrow \infty u$

$$
\Delta \mu^{2}(x) \geqslant 0, \quad 0<|\nabla \mu| \leqslant \text { const }
$$

то при выполнении условий (3) и условия $A$ оператор $M$ (1) (2) самосопряжен в существенном. 
ДоКАЗАТЕЛЬСТВо является непосредственной проверкой условий теоремы $1 \mathrm{c} k=1$, $B(x)=I_{n}$.

Пусть связная область $G \subseteq \mathbb{R}^{n}$ является полным римановьм многообразием с метрическим тензором $B^{-1}(x)$, где симметрическая матрица-функция $B(x)>0$. Рассмотрим семейство геодезических, проходящих через некоторую точку $x_{0}$. Пусть эти геодезические не имеют точек пересечения (кроме $x_{0}$ ). Они покрьвают область $G$ (см. [8, с. 167 , теорема 4.2]). Если $s$ - канонический параметр на геодезической, то в области $G$ можно определить векторное поле

$$
\vec{p}(x)=\left.\dot{x}_{s}\right|_{x(s)=x},
$$

где $x(s)$ - геодезическая семейства, проходящая через точку $x$. Это векторное поле будем назьвать иентральным геодезическим потоком, исходящим из точки $x_{0}$. Обозначим также через $r(x)$ расстояние точки $x$ до $x_{0}$ в метрике риманова многообразия.

СлЕДСТвИЕ 3. Пусть связная область $G$ является полныц римановым многообразием с метрическим тензором $B^{-1}(x)$, әде позитивная матрица-функиия $B(x) \in C^{1}(G)$. Пусть также существует исходящий из некоторой точки $x_{0}$ чентральный геодезический поток $\vec{p}(x) \in C^{1}(G)$, удовлетворяющий неравенству

$$
\nabla \vec{p}(x) \geqslant-\frac{1}{r(x)}, \quad x \in G \backslash\left\{x_{0}\right\}
$$

Eсли в операторе $M$ матрица $A(x)$ при $r(x) \geqslant N>0$ имеет вид

$$
A(x)=a(r(x)) B(x),
$$

әде $0<a(\cdot) \in C^{1}([0 ; \infty))$, то при выполнении условий (3) и условия $A$ оператор $M$ (1) (2) самосопряжен в существенном.

ДокАЗАТЕЛЬСтво. Заметим, что $\left(B^{-1} \vec{p}, \vec{p}\right)=1$, а также то, что выражение $\left(B^{-1} \vec{p}\right.$, $\overrightarrow{d x}$ ), где $\overrightarrow{d x}=\left\{d x_{1}, d x_{2}, \ldots, d x_{n}\right\}$, является полньм дифференциалом функции $r(x)$ (см. $\left[9\right.$, c. 29-51]). Воспользуемся частньм случаем теоремы 1 при $k=1, \mu_{1}(x)=\mu(x)=r(x)$, $B_{1}(x)=B(x)$. Тогда

$$
\begin{gathered}
B \nabla \mu^{2}=B \nabla r^{2}=2 r \cdot B \nabla r=2 r B \cdot B^{-1} \vec{p}=2 r \vec{p}, \\
\nabla\left(B \nabla \mu^{2}\right)=2 \nabla(r \vec{p})=2(\nabla r, \vec{p})+2 r \nabla \vec{p}=2\left(B^{-1} \vec{p}, \vec{p}\right)+2 r \nabla \vec{p}=2(1+r \nabla \vec{p}) .
\end{gathered}
$$

В силу условия (10) выполнено условие (8) теоремы 1. Далее,

$$
(B \nabla \mu, \nabla \mu)=(B \nabla r, \nabla r)=\left(B^{-1} \vec{p}, \vec{p}\right)=1 .
$$

Поэтому и условие (9) тоже вьполнено. Следствие 3 доказано.

Поскольку условие (10) является трудно проверяемым, вопрос о том, содержит ли следствие 3 случай $G \neq \mathbb{R}^{n}$ без дополнительных рассмотрений решить нельзя. Следуюший простой пример показывает, что в теореме 1 этот случай содержится. 
СЛЕДСТвИЕ 4. Пусть область $G$ является полосой в $\mathbb{R}^{2}$ :

$$
G=\left\{x \in \mathbb{R}^{2} ;-\infty<x_{1}<\infty ;-h<x_{2}<h\right\},
$$

в которой задана функиия $\mu(x)=\sqrt{x_{1}^{2}-h^{2} \ln \left(1-x_{2}^{2} / h^{2}\right)^{\alpha}}$ с константой $\alpha \in$ $(0,1 / 2]$. Если при $\mu(x) \geqslant N>0$ матрича $A(x)$ в операторе $M$ имеет вид

$$
A(x)=a(\mu)\left(\begin{array}{cc}
1 & 0 \\
0 & \left(1-x_{2}^{2} / h^{2}\right)^{2}
\end{array}\right),
$$

әде $0<a(\cdot) \in C^{1}([0, \infty))$, то при выполнении условий (3) и условия $A$ оператор $M(1),(2)$ самосопряжен в существенном.

ДокАЗАТЕЛЬСтво. Проверим вьполнение условий (8), (9) теоремы $1 \mathrm{c} k=1$, $\mu_{1}(x)=\mu(x)$,

$$
B_{1}(x)=B(x)=\left(\begin{array}{cc}
1 & 0 \\
0 & \left(1-x_{2}^{2} / h^{2}\right)^{2}
\end{array}\right) .
$$

Имеем

$$
\nabla\left(B \nabla \mu^{2}\right)=2(1+\alpha)-6 \alpha \frac{x_{2}^{2}}{h^{2}} \geqslant 2-4 \alpha \geqslant 0,
$$

т.е. условие (8) выполняется. Далее,

$$
(B \nabla \mu, \nabla \mu)=\frac{x_{1}^{2}+\alpha^{2} x_{2}^{2}}{x_{1}^{2}-h^{2} \ln \left(1-x_{2}^{2} / h^{2}\right)^{\alpha}} .
$$

Поскольку при $0 \leqslant t<1$

получаем

$$
-\ln (1-t)=t+\frac{t^{2}}{2}+\frac{t^{3}}{3}+\cdots \geqslant t
$$

$$
(B \nabla \mu, \nabla \mu) \leqslant \frac{x_{1}^{2}+\alpha^{2} x_{2}^{2}}{x_{1}^{2}+\alpha x_{2}^{2}} \leqslant 1 .
$$

То есть условие (9) тоже выполняется. Следствие 4 доказано.

4. Накрывающие семейства и самосопряженность в существенном оператора $M$. Назовем семейство функций $\psi(x, \tau)$, определенных в $G$, с параметром $\tau \in$ $\left[\tau_{0}, \infty\right)$, накрыввающим область $G$, если оно удовлетворяет следующим условиям:

1c) $0 \leqslant \psi(x, \tau) \in C\left(G \times\left[\tau_{0}, \infty\right)\right)$ и при любом $\tau \in\left[\tau_{0}, \infty\right)$

$$
\psi(x, \tau) \in C_{0}(G) \cap C^{(1,1)}(\operatorname{supp} \psi) ;
$$

2 c) открытые множества $\Omega_{\tau}=\operatorname{Int} \operatorname{supp} \psi(x, \tau)\left(\bar{\Omega}_{\tau}=\operatorname{supp} \psi\right)$ расширяются с ростом $\tau$, т.е. $\tau_{2}>\tau_{1} \Rightarrow \bar{\Omega}_{\tau_{1}} \subset \Omega_{\tau_{2}}$. При этом для любого компакта $R \subset G$ при достаточно больших $\tau R \subset \Omega_{\tau}$;

$3 c)$ при любом $\tau \in\left[\tau_{0}, \infty\right) \quad \partial \Omega_{\tau}$ состоит из конечного числа кусочно гладких замкнутых гиперповерхностей, причем для любой точки $x \in G \backslash \Omega_{\tau_{0}}$ существует единственное значение $\tau$, при котором $x \in \partial \Omega_{\tau}$;

4c) условие $3 \mathrm{c}$ ) определяет функцию

$$
\tau=\rho(x) \in \operatorname{Lip}_{1 \mathrm{loc}}\left(G \backslash \Omega_{\tau_{0}}\right) ;
$$

5c) при каждом $x \in G$

$$
\int_{\tau_{0}}^{\infty} \psi(x, \tau) d \tau=\infty
$$


В дальнейшем используется вектор-функция $\left.\left(\nabla_{x} \psi\right)\right|_{\tau=\rho(x)}$, которая понимается в смысле предельных значений $\nabla_{x} \phi$ на $\partial \Omega_{\tau}$ из внутренности области $\Omega_{\tau}$.

Основным результатом является

ТЕОрема 2. Пусть для оператора $M$ выполнены условия (3) и условие $A$, а также существует накрывающее область $G$ семейство, с которым матрица $A(x)$ старших коэффичиентов оператора $M$ удовлетворяет неравенствам

$$
\begin{gathered}
\nabla_{x}\left(A \nabla_{x} \psi\right) \leqslant C \psi \quad \text { почти всюду в } \Omega_{\tau}\left(\tau \geqslant \tau_{0}\right), \\
\left(\left.A\left(\nabla_{x} \psi\right)\right|_{\tau=\rho(x)}, \nabla \rho\right) \geqslant-C \quad \text { почти всюду в } G \backslash \Omega_{\tau_{0}}
\end{gathered}
$$

с некоторой константой $C>0$. Тогда оператор $M$ вида (1), (2) самосопряжен в существенном.

Для доказательства теоремы 2 потребуются две леммы о свойствах накрывающих семейств.

ЛЕмма 1. Для любого компакта $R \subset G$ и любого числа $N>0$ найдется такое $\tau_{N}>0$, для которого при всех $x \in R \backslash \Omega_{\tau_{0}}$ выполнено неравенство

$$
\int_{\rho(x)}^{\tau_{N}} \psi(x, \tau) d \tau \geqslant N
$$

ДоКАЗАТЕЛЬСТВо. Из условия 5с) следует, что для всякой точки $x_{0} \in G \backslash \Omega_{\tau_{0}}$ и любого $N>0$ найдется $\tau_{N}^{0}>0$, для которого

$$
\int_{\rho\left(x_{0}\right)}^{\tau_{N}^{0}} \psi\left(x_{0}, \tau\right) d \tau \geqslant N+2
$$

В силу условия 1 c) $\psi(x, \tau)$ равномерно непрерьвна в каждом цилиндре $Z_{\varepsilon}=\bar{U}_{\varepsilon} \times$ $\left[\tau_{0} ; \tau_{N}^{0}\right]$, где $U_{\varepsilon}-$ окрестность точки $x_{0}\left(\bar{U}_{\varepsilon} \subset G\right)$ радиуса $\varepsilon>0$. Поэтому в силу условия 4c) можно выбрать $\varepsilon>0$ так, чтобы при $y \in U_{\varepsilon} \backslash \Omega_{\tau_{0}}$ были справедливы неравенства

$$
\max _{(x, \tau) \in Z_{\varepsilon}} \psi(x, \tau) \cdot\left|\rho(y)-\rho\left(x_{0}\right)\right| \leqslant 1, \quad \psi(y, \tau) \geqslant \psi\left(x_{0}, \tau\right)-\frac{1}{\tau_{N}^{0}}, \quad \tau \in\left[\tau_{0}, \tau_{N}^{0}\right] .
$$

Отсюда следует, что при $x \in U \varepsilon \backslash \Omega_{\tau_{0}}$

$$
\begin{aligned}
\int_{\rho(x)}^{\tau_{N}^{0}} \psi(x, \tau) d \tau & \geqslant \int_{\rho\left(x_{0}\right)}^{\tau_{N}^{0}} \psi(x, \tau) d \tau-\left|\int_{\rho\left(x_{0}\right)}^{\rho(x)} \psi(x, \tau) d \tau\right| \\
& \geqslant \int_{\rho\left(x_{0}\right)}^{\tau_{N}^{0}} \psi\left(x_{0}, \tau\right) d \tau-\frac{\tau_{N}^{0}-\rho\left(x_{0}\right)}{\tau_{N}^{0}}-1 \geqslant N .
\end{aligned}
$$

Таким образом, для любой точки $x_{0}$ найдется окрестность $U_{\varepsilon}$ и число $\tau_{N}^{0}>0$, для которого

$$
\int_{\rho(x)}^{\tau_{N}^{0}} \psi(x, \tau) d \tau \geqslant N, \quad x \in U_{\varepsilon} \backslash \Omega_{\tau_{0}} .
$$

Если для каждой точки множества $R \backslash \Omega_{\tau_{0}}$ взять такую окрестность, то они составят покрытие, из которого можно выделить конечное. Это даст нам числа $\tau_{N j}^{0}(j=1,2, \ldots, k)$. Очевидно, что при $\tau_{N}=\max _{j} \tau_{N j}^{0}$ выполнено неравенство (13). Лемма 1 доказана. 
Лемма 2. Пусть оператор $M$ вида (1) удовлетворяет условию $A$, а $\psi=\psi(x, \tau)-$ накрывающее область $G$ семейство. Тогда для любого $u \in D_{\text {lос }}\left(M^{*}\right)$ и любого $t>\tau_{0}$ найдется такая последовательность $\left\{u_{m}\right\}_{m=1}^{\infty}$ функиий из $C^{2}(G)$, что $u_{m} \rightarrow u$ в $L_{2}\left(\Omega_{t}\right)$, а последовательность функиий $C_{m}(\tau)=\operatorname{Re}\left\langle\psi M u_{m}, u_{m}\right\rangle_{L_{2}}\left(\Omega_{\tau}\right)$ сходится к функиии $C(\tau)=\operatorname{Re}\langle\psi g, u\rangle_{L_{2}\left(\Omega_{\tau}\right)}$ равномерно по $\tau \in\left[\tau_{0}, t\right]$.

ДокАЗАтЕльство. Пусть ограниченная область $\Omega$ такова, что $\bar{\Omega}_{t} \subset \Omega$. Согласно условию А должна существовать последовательность функций $u_{m} \in C^{2}(G)$, сходящаяся к $u$ в $L_{2}(\Omega)$, а значит и в $L_{2}\left(\Omega_{t}\right)$. Нужно доказать, что последовательность функций $C_{m}(\tau)=\operatorname{Re}\left\langle\psi M u_{m}, u_{m}\right\rangle$ сходится к функции $C(\tau)=\operatorname{Re}\langle\psi g, u\rangle$ равномерно по $\tau \in\left[\tau_{0}, t\right]$. Для этого заметим, что

$$
J_{m}[\phi]=\operatorname{Re}\left\langle\phi M u_{m}, u_{m}\right\rangle_{L_{2}(\Omega)}, \quad J[\phi]=\operatorname{Re}\langle\phi g, u\rangle_{L_{2}(\Omega)}
$$

являются ограниченными линейными функционалами в вещественном банаховом пространстве $C_{0}(\Omega)$ с нормой

$$
\|\phi\|_{C_{0}(\Omega)}=\max _{x \in \Omega}|\phi(x)| .
$$

Поскольку при каждом $\phi \in C_{0}(\Omega)$

$$
\lim _{m \rightarrow \infty} J_{m}[\phi]=J[\phi]
$$

по теореме Банаха-Штейнхауза последовательность норм функционалов $J_{m}[\phi]$ ограничена, т.е. при некоторой константе $K>0$

$$
|J[\phi]|,\left|J_{m}[\phi]\right| \leqslant K\|\phi\|_{C_{0}(\Omega)}
$$

для любой функции $\phi \in C_{0}(\Omega)$. В силу равномерной непрерывности $\psi(x, \tau)$ на $\bar{\Omega} \times\left[\tau_{0}, t\right]$ при любом $\varepsilon>0$ для $\tau^{\prime} \in\left[\tau_{0}, t\right]$ найдется такая окрестность $V_{\tau^{\prime}}$, что при $x \in \Omega$

$$
|\delta \psi|=\left|\psi\left(x, \tau^{\prime}\right)-\psi(x, \tau)\right|<\frac{\varepsilon}{3 K}, \quad \tau \in V_{\tau^{\prime}} \cap\left[\tau_{0}, t\right]
$$

Поскольку $C_{m}(\tau) \rightarrow C(\tau)$ поточечно, найдется такой номер $m_{\varepsilon}\left(\tau^{\prime}\right)$, что при $m \geqslant m_{\varepsilon}\left(\tau^{\prime}\right)$, $\tau \in V_{\tau^{\prime}} \cap\left[\tau_{0}, t\right]$

$$
\begin{aligned}
\left|C_{m}(\tau)-C(\tau)\right| & \leqslant\left|C_{m}(\tau)-C_{m}\left(\tau^{\prime}\right)\right|+\left|C\left(\tau^{\prime}\right)-C(\tau)\right|+\left|C_{m}\left(\tau^{\prime}\right)-C\left(\tau^{\prime}\right)\right| \\
& \leqslant\left|J_{m}[\delta \psi]\right|+|J[\delta \psi]|+\frac{\varepsilon}{3}<\varepsilon .
\end{aligned}
$$

Если для каждой точки $\tau^{\prime} \in\left[\tau_{0}, t\right]$ взять окрестность $V_{\tau^{\prime}}$ и выбрать из этого покрытия конечное, то получим конечное число номеров $m_{\varepsilon}\left(\tau_{j}^{\prime}\right)$. При $m \geqslant m_{\varepsilon}=\max _{j} m_{\varepsilon}\left(\tau_{j}^{\prime}\right)$

$$
\left|C_{m}(\tau)-C(\tau)\right|<\varepsilon, \quad \tau \in\left[\tau_{0}, t\right]
$$

Лемма 2 доказана. 
ДОКАЗАТЕЛЬСТВО ТЕОРЕМЫ 2. Не ограничивая общности можно считать, что при $x \in G$

$$
q(x) \geqslant \frac{1}{2} C+1
$$

где $C$ - константа в условиях $(11),(12)$. Достаточно показать, что при условии (14)

$$
M^{*} u=0 \Rightarrow u(x) \equiv 0 \quad \text { при } x \in G \backslash \Omega_{\tau_{0}} .
$$

Действительно, с помощью интегрирования по частям нетрудно установить, что для вещественной функции $\phi(x) \in C_{0}^{\infty}(G)$ и любой функции $v(x) \in C^{2}(G)$ справедливо равенство

$$
\langle M(\phi v), \phi v\rangle_{L_{2}(G)}=\int_{G}(A \nabla \phi, \nabla \phi)|v|^{2} d x+\operatorname{Re}\left\langle\phi^{2} M v, v\right\rangle_{L_{2}(G)}
$$

Откуда, учитывая (14), получаем

$$
\|\phi v\|_{L_{2}(G)}^{2} \leqslant \int_{G}(A \nabla \phi, \nabla \phi)|v|^{2} d x+\operatorname{Re}\left\langle\phi^{2} M v, v\right\rangle_{L_{2}(G)} .
$$

Выбирая в этом неравенстве $v=u_{m}(x)$, где $\left\{u_{m}\right\}$ - последовательность из условия А, отвечающая области $\Omega_{\tau}$ такой, что $\operatorname{supp} \phi \subset \Omega_{\tau}$, и переходя к пределу при $m \rightarrow \infty$, получим

$$
\|\phi u\|^{2} \leqslant \int_{G}(A \nabla \phi, \nabla \phi)|u|^{2} d x .
$$

Если $u(x) \equiv 0$ при $x \in G \backslash \Omega \tau_{0}$, а функция $\phi(x) \equiv 1$ при $x \in \Omega_{\tau_{0}}$, то $u(x) \equiv 0$ при $x \in G$. Последнее влечет самосопряженность оператора $\bar{M}$.

Для доказательства справедливости утверждения (15) рассмотрим получаемое интегрированием по частям равенство

$$
\begin{array}{r}
\int_{\Omega_{\tau}} \psi(A(\nabla-i \vec{b}) v,(\nabla-i \vec{b}) v) d x+\int_{\Omega_{\tau}} \psi q|v|^{2} d x \\
=\operatorname{Re} \int_{\Omega_{\tau}} \psi \bar{v} M v d x-\frac{1}{2} \int_{\Omega_{\tau}}\left(A \nabla \psi, \nabla|v|^{2}\right) d x
\end{array}
$$

где $\psi=\psi(x, \tau)$ - функция накрывающего семейства, а $v(x)$ - произвольная функция из $C^{2}(G)$. Учитьвая равенство

$$
\frac{1}{2} \int_{\Omega_{\tau}}\left(A \nabla \psi, \nabla|v|^{2}\right) d x=-\frac{1}{2} \int_{\Omega_{\tau}} \nabla(A \nabla \psi) \cdot|v|^{2} d x+\frac{1}{2} \int_{\partial \Omega_{\tau}}|v|^{2}(A \nabla \psi, \overrightarrow{d S}),
$$

а также позитивность матрицы $A(x)$, получим неравенство

$$
\int_{\Omega_{\tau}} \psi q|v|^{2} d x \leqslant \frac{1}{2} \int_{\Omega_{\tau}} \nabla(A \nabla \psi) \cdot|v|^{2} d x-\frac{1}{2} \int_{\partial \Omega_{\tau}}|v|^{2}(A \nabla \psi, \overrightarrow{d S})+\operatorname{Re} \int_{\Omega_{\tau}} \psi \bar{v} M v d x
$$

Отсюда в силу условия (11) и неравенства (14) следует неравенство

$$
\int_{\Omega_{\tau}} \psi|v|^{2} d x \leqslant-\frac{1}{2} \int_{\partial \Omega_{\tau}}|v|^{2}(A \nabla \psi, \overrightarrow{d S})+\operatorname{Re} \int_{\Omega_{\tau}} \psi \bar{v} M v d x
$$


Проинтегрируем последнеенеравенство по $\tau$ в пределах от $\tau_{0}$ до $t$, учитывая, что $\overrightarrow{d S} d \tau=$ $\nabla \rho d x$. Получим неравенство

$$
\begin{aligned}
\int_{\Omega_{t} \backslash \Omega_{\tau_{0}}}\left(\int_{\rho(x)}^{t} \psi(x, \tau) d \tau\right)|v|^{2} d x \leqslant & -\frac{1}{2} \int_{\Omega_{t} \backslash \Omega_{\tau_{0}}}\left(\left.A(\nabla \psi)\right|_{\tau=\rho(x)}, \nabla \rho\right)|v|^{2} d x \\
& +\int_{\tau_{0}}^{t}\left(\operatorname{Re} \int_{\Omega_{\tau}} \psi \bar{v} M v d x\right) d \tau .
\end{aligned}
$$

Поскольку условие А считается вьполненным, в последнем неравенстве в качестве $v$ можно взять элементы последовательности $\left\{u_{m}\right\}$, соответствующей $u \in L_{2}(G)$ такому, что $M^{*} u=0$.

Переходя к пределу при $m \rightarrow \infty$ и учитьвая лемму 2, получим

$$
\int_{\Omega_{t} \backslash \Omega_{\tau_{0}}}\left(\int_{\rho(x)}^{t} \psi(x, \tau) d \tau\right)|u|^{2} d x \leqslant-\frac{1}{2} \int_{\Omega_{t} \backslash \Omega_{\tau_{0}}}\left(\left.A(\nabla \psi)\right|_{\tau=\rho(x)}, \nabla \rho\right)|u|^{2} d x .
$$

В силу условия (12) выполнено неравенство

$$
\int_{\Omega_{t} \backslash \Omega_{\tau_{0}}}\left(\int_{\rho(x)}^{t} \psi(x, \tau) d \tau\right)|u|^{2} d x \leqslant \frac{C}{2}\|u\|_{L_{2}(G)}^{2} .
$$

Отсюда в силу леммы 1 заключаем, что справедливо утверждение (15). Теорема доказана.

ДОКАЗАТЕЛЬСТВо ТЕОРЕМЫ 1. ДЛя построения накрывающего семейства при условиях теоремы 1 рассмотрим интегралы

$$
f_{j}^{ \pm}(z)=\int_{0}^{z} \Theta(t) a_{j}^{-1}( \pm \sqrt{t}) d t
$$

где функция $\Theta(t) \in C^{2}([0, \infty))$ такова, что $\Theta(t) \equiv 0$ при $0 \leqslant t \leqslant \tau_{0}-1, \Theta(t) \equiv 1$ при $t \geqslant \tau_{0}>1, \Theta^{\prime}(t) \geqslant 0$. Введем функции двух переменных $(j=1, \ldots, k)$

$$
\psi_{j}(s, \tau)= \begin{cases}1-\frac{f_{j}^{+}\left(s^{2}\right)}{f_{j}^{+}(\tau)}, & s \in\left(0, \tau^{1 / 2}\right], \\ 1-\frac{f_{j}^{-}\left(s^{2}\right)}{f_{j}^{-}(\tau)}, & s \in\left[-\tau^{1 / 2}, 0\right], \\ 0, & s \notin\left[-\tau^{1 / 2}, \tau^{1 / 2}\right] .\end{cases}
$$

Эти функции определены при $\tau \in\left[\tau_{0}, \infty\right), s \in \mathbb{R}^{1}$.

Пусть

$$
\psi(x, \tau)=\frac{1}{\tau} \prod_{j=1}^{n} \psi_{j}\left(\mu_{j}\left(\vec{x}_{j}, \tau\right)\right)
$$

Нетрудно проверить, что это семейство функций удовлетворяет всем условиям накрывающего область $G$ семейства, причем

$$
\Omega_{\tau}=\left\{x: \max _{j} \mu_{j}^{2}\left(\vec{x}_{j}\right)<\tau\right\}, \quad \rho(x)=\max _{j} \mu_{j}^{2}\left(\vec{x}_{j}\right) \in \operatorname{Lip}_{1 \mathrm{loc}}\left(G \backslash \Omega_{\tau_{0}}\right) .
$$


Отметим, что число $\tau_{0}$ можно выбрать столь большим, чтобы при каждом $j=1,2, \ldots, k$

$$
Q_{j} \subset\left\{\vec{x}_{j}: \mu_{j}^{2}\left(\vec{x}_{j}\right)<\tau_{0}-1\right\} .
$$

Поэтому при $x \in Q, \tau \geqslant \tau_{0} \quad \psi(x, \tau) \equiv 1$, что означает справедливость условий теоремы 2 при $x \in Q$. Если же $\vec{x}_{j} \in G_{j} \backslash Q_{j}$, то

$$
\begin{aligned}
a_{j}\left(\mu_{j}\right) B_{j}\left(\vec{x}_{j}\right) \nabla f_{j}^{ \pm}\left(\mu_{j}^{2}\left(\vec{x}_{j}\right)\right)= & \Theta\left(\mu_{j}^{2}\left(\vec{x}_{j}\right)\right) \cdot B_{j}\left(\vec{x}_{j}\right) \nabla \mu_{j}^{2}\left(\vec{x}_{j}\right), \\
\nabla\left(a_{j}\left(\mu_{j}\right) B_{j} \nabla f_{j}^{ \pm}\left(\mu_{j}^{2}\left(\vec{x}_{j}\right)\right)\right)= & \Theta^{\prime}\left(\mu_{j}^{2}\left(\vec{x}_{j}\right)\right) \cdot\left(B_{j}\left(\vec{x}_{j}\right) \nabla \mu_{j}^{2}, \nabla \mu_{j}^{2}\right) \\
& +\Theta\left(\mu_{j}^{2}\left(\vec{x}_{j}\right)\right) \cdot \nabla\left(B_{j}\left(\vec{x}_{j}\right) \nabla \mu_{j}^{2}\left(\vec{x}_{j}\right)\right) \geqslant 0 .
\end{aligned}
$$

Отсюда следует, что

$$
\nabla\left(a_{j}\left(\mu_{j}\right) B_{j}\left(\vec{x}_{j}\right) \cdot \nabla \psi_{j}\left(\mu_{j}, \tau\right)\right) \leqslant 0 .
$$

Последнее, очевидно, влечет справедливость условия (11) теоремы 2.

Если считать $\nabla \mu_{j}$ и $B_{j} \nabla \mu_{j} n$-компонентными векторами вида $\left\{0, \ldots, 0, \vec{y}_{j}, 0, \ldots, 0\right\}$, то можно написать равенство

$$
\left.A(\nabla \psi)\right|_{\tau=\rho(x)}=\sum_{j=1}^{k} \frac{\mu_{j}\left(\vec{x}_{j}\right)}{\rho(x)} \Phi_{j}(x) B_{j}\left(\vec{x}_{j}\right) \nabla \mu_{j}\left(\vec{x}_{j}\right),
$$

где скалярные функции $\Phi_{j}(x)$ ограничены в $G \backslash Q$. Определенньй почти всюду вектор $\nabla \rho$ равен $\nabla \mu_{j_{0}}^{2}\left(\vec{x}_{j_{0}}\right)$ при почти каждом $x$ с зависящим от него $j_{0}$. Следовательно, почти всюду в $G \backslash Q$ справедливо неравенство

$$
\left|\left(\left.A(\nabla \psi)\right|_{\tau=\rho(x)}, \nabla \rho\right)\right| \leqslant \mathrm{const} \sum_{j=1}^{k}\left(B_{j} \nabla \mu_{j}, \nabla \mu_{j}\right),
$$

которое в силу условия (9) влечет справедливость (12). Теорема 1 доказана.

\section{СПИСОК ЦИТИРОВАННОЙ ЛИТЕРАТУРЫ}

[1] Weyl H. Über gewöhnliche Differentialgleichungen mit Singularitäten und die zugehörigen Entwicklungen willkürlicher Funktionen // Math. Ann. 1910. V. 68. P. 222-269.

[2] Уральцева Н.Н. О несамосопряженности в $L_{2}\left(\mathbb{R}^{n}\right)$ эллиптического оператора с быстро растущими коэффициентами // Зап. науч. семинаров ЛОМИ АН СССР. 1969. Т. 149. C. 288-294.

[3] Лаптев С. А. О замыкании в метрике обобщенного интеграла Дирихле // Дифференц. уравнения. 1971. Т. 7. № 4. С. 727-736.

[4] Devinatz A. Essential self-adjointness of Schrödinger-type operators // J. Funct. Anal. 1977. V. 25. № 1. P. 58-69.

[5] Рофе-Бекетов Ф. С. Замечание в связи с многомерным обобщением теоремы Г. Вейля о самосопряженности // Теория функций, функцион. анализ и их приложения. 1989. № 52. С. 88-90.

[6] Стейн И. Сингулярные интегралы и дифференциальные свойства функций. М.: Мир, 1972.

[7] Kato T. Schrödinger operators with singular potentials // Israel J. Math. 1972. V. 13. P. $135-148$.

[8] Кобаяси Ш., Номидзу К. Основы дифференциальной геометрии. Т. 1. М.: Наука, 1981.

[9] Ахиезер Н. И. Вариационное исчисление. Харьков: Вища школа, 1981. 